\title{
Directed flow as effect of transient matter rotation in hadron and nucleus collisions
}

\author{
S.M. Troshin, N.E. Tyurin \\ Institute for High Energy Physics, \\ Protvino, Moscow Region, 142281, Russia
}

\begin{abstract}
We discuss directed flow $v_{1}$, observable introduced for description of nucleus collisions. We consider its possible origin in hadronic reactions as a result of rotation of the transient matter and trace analogy with nucleus collisions.
\end{abstract}




\section{Introduction}

Multiparticle production in hadron and nucleus collisions and corresponding observables provide a clue to the mechanisms of confinement and hadronization. Discovery of the deconfined state of matter has been announced by the four major experiments at RHIC [1]. Despite the highest values of energy and density have been reached, a genuine quark-gluon plasma QGP (gas of the free current quarks and gluons) was not found 1 . The deconfined state reveals the properties of the perfect liquid, being strongly interacting collective state and therefore it was labelled as sQGP [3]. The nature of new form of matter discovered is not known and variety of models has been proposed to treat its properties [4]. The importance of the experimental discoveries at RHIC is that the matter is strongly correlated and reveals high degree of the coherence when it is well beyond the critical values of density and temperature.

Important tools in the studies of the nature of the new form of matter are the anisotropic flows which are the quantitative characteristics of the collective motion of the produced hadrons in the nuclear interactions. With their measurements one can obtain a valuable information on the early stages of reactions and observe signals of QGP formation [5, 6, 7, 8, 9, 10, 11, 12, 13].

The measurements of anisotropic flows and constituent quark scaling demonstrated an importance of the constituent quarks [14] and their role as effective degrees of freedom of the newly discovered state of matter.

The recent review paper [15] provides an emphasis on the historic aspects of the QGP searches. The important conclusion made in this work is that the deconfined state of matter has being observed in hadronic reactions during a long time and it would be interesting to study collective properties of transient state in reactions with hadrons and nuclei simultaneously. Thus, keeping in mind that the dynamics of strong interactions is the same for the hadron and nucleus collisions, the following question naturally arises, namely, what, if anything, the recent discoveries at RHIC implement for hadronic interactions?

In this note we try to address in a model way one aspect of this broad problem, i.e. we discuss the role of the coherent rotation of the transient matter in hadron collisions as the origin of the directed flow in these reactions and stress that behavior of collective observable $v_{1}$ in hadronic and nuclear reactions would be similar.

The already mentioned experimental probes of collective dynamics in $A A$ interactions [16, 17], the momentum anisotropies $v_{n}$ are defined by means of the Fourier expansion of the transverse momentum spectrum over the momentum az-

\footnotetext{
${ }^{1}$ It is to be noted here that confinement due to causality principle might exclude the very existence of QGP defined that way [2].
} 
imuthal angle $\phi$. The angle $\phi$ is the angle of the detected particle transverse momentum with respect to the reaction plane spanned by the collision axis $z$ and the impact parameter vector $\mathbf{b}$ directed along the $x$ axis. Thus, the anisotropic flows are the azimuthal correlations with the reaction plane. In particular, the directed flow is defined as

$$
v_{1}\left(p_{\perp}\right) \equiv\langle\cos \phi\rangle_{p_{\perp}}=\left\langle p_{x} / p_{\perp}\right\rangle=\left\langle\hat{\mathbf{b}} \cdot \mathbf{p}_{\perp} / p_{\perp}\right\rangle
$$

From Eq. (1) it is evident that this observable can be used for studies of multiparticle production dynamics in hadronic collisions provided that impact parameter $\mathbf{b}$ is fixed. Therefore we discuss hereabout directed flow $v_{1}$ in hadron collisions at fixed impact parameters and refer after that those considerations to the collisions of nuclei proceeding from the same nature of the transient states in both cases. It is reasonable to proceed that way in the framework of the constituent quark model picture where hadron structure looks similar to the structure of light nuclei. In particular, we amend the model [18, 19] developed for hadron interactions (based on the chiral quark model ideas) and consider the effect of collective rotation of a quark matter in the overlap region. We formulate a hypothesis on connection of the strongly interacting transient matter rotation with the directed flow generation.

The outline of the paper is the following. In section 1 the dynamics of hadronic interaction, nature of transient state and effective degrees of freedom of the transient state are treated. Section 2 is devoted to the specific role of the transient matter rotation as an origin of the directed flow in hadronic reactions. The energy, rapidity and transverse momentum dependencies are discussed there. Section 3 considers similarity and difference of nuclei with hadron collisions and discussion of the recent experimental measurements of the directed flow at RHIC. In the final section we discuss possible relation of the directed flow with the nature of transient matter, i.e. is the matter strongly interacting or a weakly interacting one at the LHC energies and provide a conclusion.

\section{Effective degrees of freedom and transient state of matter in hadron collisions}

We would like to point out the possibility that the origin of the transient state and its dynamics along with hadron structure can be related to the mechanism of spontaneous chiral symmetry breaking $(\chi \mathrm{SB})$ in QCD [20], which leads to the generation of quark masses and appearance of quark condensates. This mechanism describes transition of the current into constituent quarks. The gluon field is considered to be responsible for providing quarks with masses and its internal structure through the instanton mechanism of the spontaneous chiral symmetry 
breaking. Massive constituent quarks appear as quasiparticles, i.e. current quarks and the surrounding clouds of quark-antiquark pairs which consist of a mixture of quarks of the different flavors. Quark radii are determined by the radii of the surrounding clouds. Quantum numbers of the constituent quarks are the same as the quantum numbers of current quarks due to conservation of the corresponding currents in QCD.

Collective excitations of the condensate are the Goldstone bosons and the constituent quarks interact via exchange of the Goldstone bosons; this interaction is mainly due to pion field. Pions themselves are the bound states of massive quarks. The interaction responsible for quark-pion interaction can be written in the form [21]:

$$
\mathcal{L}_{I}=\bar{Q}\left[i \not \partial-M \exp \left(i \gamma_{5} \pi^{A} \lambda^{A} / F_{\pi}\right)\right] Q, \quad \pi^{A}=\pi, K, \eta .
$$

The interaction is strong, the corresponding coupling constant is about 4 . The general form of the total effective Lagrangian $\left(\mathcal{L}_{Q C D} \rightarrow \mathcal{L}_{\text {eff }}\right)$ relevant for description of the non-perturbative phase of QCD includes the three terms [22]

$$
\mathcal{L}_{\text {eff }}=\mathcal{L}_{\chi}+\mathcal{L}_{I}+\mathcal{L}_{C}
$$

Here $\mathcal{L}_{\chi}$ is responsible for the spontaneous chiral symmetry breaking and turns on first.

To account for the constituent quark interaction and confinement the terms $\mathcal{L}_{I}$ and $\mathcal{L}_{C}$ are introduced. The $\mathcal{L}_{I}$ and $\mathcal{L}_{C}$ do not affect the internal structure of the constituent quarks.

The picture of a hadron consisting of constituent quarks embedded into quark condensate implies that overlapping and interaction of peripheral clouds occur at the first stage of hadron interaction. The interaction of the condensate clouds assumed to of the shock-wave type, this condensate clouds interaction generates the quark-pion transient state. This mechanism is inspired by the shock-wave production process proposed by Heisenberg [23] long time ago. At this stage, part of the effective lagrangian $\mathcal{L}_{C}$ is turned off (it is turned on again in the final stage of the reaction). Nonlinear field couplings transform then the kinetic energy to internal energy [23, 24]. As a result the massive virtual quarks appear in the overlapping region and transient state of matter is generated. This state consist of $\bar{Q} Q$ pairs and pions strongly interacting with quarks.

Part of hadron energy carried by the outer condensate clouds being released in the overlap region goes to generation of massive quarks interacting by pion exchange and their number was estimated as follows as follows:

$$
\tilde{N}(s, b) \propto \frac{\left(1-\left\langle k_{Q}\right\rangle\right) \sqrt{s}}{m_{Q}} D_{c}^{h_{1}} \otimes D_{c}^{h_{2}} \equiv N_{0}(s) D_{C}(b),
$$


where $m_{Q}$ - constituent quark mass, $\left\langle k_{Q}\right\rangle$ - average fraction of hadron energy carried by the constituent valence quarks. Function $D_{c}^{h}$ describes condensate distribution inside the hadron $h$ and $b$ is an impact parameter of the colliding hadrons. Thus, $\tilde{N}(s, b)$ quarks appear in addition to $N=n_{h_{1}}+n_{h_{2}}$ valence quarks.

The generation time of the transient state $\Delta t_{t s g}$ in this picture obeys to the inequality

$$
\Delta t_{t s g} \ll \Delta t_{\text {int }},
$$

where $\Delta t_{\text {int }}$ is the total interaction time. The newly generated massive virtual quarks play a role of scatterers for the valence quarks in elastic scattering; those quarks are transient ones in this process: they are transformed back into the condensates of the final hadrons.

Under construction of the model for elastic scattering [18] it was assumed that the valence quarks located in the central part of a hadron are scattered in a quasiindependent way off the transient state with interaction radius of valence quark determined by its inverse mass:

$$
R_{Q}=\kappa / m_{Q} .
$$

The elastic scattering $S$-matrix in the impact parameter representation is written in the model in the form of linear fractional transform:

$$
S(s, b)=\frac{1+i U(s, b)}{1-i U(s, b)}
$$

where $U(s, b)$ is the generalized reaction matrix, which is considered to be an input dynamical quantity similar to an input Born amplitude and related to the elastic scattering scattering amplitude through an algebraic equation which enables one to restore unitarity [25]. The function $U(s, b)$ is chosen in the model as a product of the averaged quark amplitudes

$$
U(s, b)=\prod_{Q=1}^{N}\left\langle f_{Q}(s, b)\right\rangle
$$

in accordance with assumed quasi-independent nature of the valence quark scattering. The essential point here is the rise with energy of the number of the scatterers like $\sqrt{s}$. The $b$-dependence of the function $\left\langle f_{Q}\right\rangle$ has a simple form $\left\langle f_{Q}(b)\right\rangle \propto \exp \left(-m_{Q} b / \xi\right)$.

These notions can be extended to particle production with account of the geometry of the overlap region and dynamical properties of the transient state. Valence constituent quarks would excite a part of the cloud of the virtual massive quarks and those quarks will subsequently hadronize and form the multiparticle final state. This mechanism can be relevant for the region of moderate transverse 
momenta while the region of high transverse momenta should be described by the excitation of the constituent quarks themselves and application of the perturbative QCD to the parton structure of the constituent quark. The model allow to describe elastic scattering and the main features of multiparticle production [18, 19, 26]. In particular, it leads to asymptotical dependencies

$$
\sigma_{\text {tot }, e l} \sim \ln ^{2} s, \quad \sigma_{\text {inel }} \sim \ln s, \quad \bar{n} \sim s^{\delta} .
$$

Inclusive cross-section for unpolarized particles integrated over impact parameter $\mathbf{b}$, cannot depend on the azimuthal angle of the detected particle transverse momentum. The $s$-channel unitarity for the inclusive cross-section could be accounted for by the following representation

$$
\frac{d \sigma}{d \xi}=4 \int_{0}^{\infty} d \mathbf{b} \frac{I(s, \mathbf{b}, \xi)}{|1-i U(s, \mathbf{b})|^{2}}
$$

The set of kinematic variables denoted by $\xi$ describes the state of the detected particle. The function $I$ is constructed from the multiparticle analogs $U_{n}$ of the function $U$ and is in fact an non-unitarized inclusive cross-section in the impact parameter space and unitarity corrections is given by the factor

$$
w(s, b) \equiv|1-i U(s, b)|^{-2}
$$

in Eq. (8). Unitarity modifies anisotropic flows. When the impact parameter vector $\mathbf{b}$ and transverse momentum $\mathbf{p}_{\perp}$ of the detected particle are fixed, the function $I=\sum_{n \geq 3} I_{n}$, where $n$ denotes a number of particles in the final state, depends on the azimuthal angle $\phi$ between vectors $\mathbf{b}$ and $\mathbf{p}_{\perp}$. It should be noted that the impact parameter $\mathbf{b}$ is the variable conjugated to the transferred momentum $\mathbf{q} \equiv \mathbf{p}_{a}^{\prime}-\mathbf{p}_{a}$ between two incident channels which describe production processes of the same final multiparticle state. The dependence on the azimuthal angle $\phi$ can be written in explicit form through the Fourier series expansion

$$
I\left(s, \mathbf{b}, y, \mathbf{p}_{\perp}\right)=I_{0}\left(s, b, y, p_{\perp}\right)\left[1+\sum_{n=1}^{\infty} 2 \bar{v}_{n}\left(s, b, y, p_{\perp}\right) \cos n \phi\right] .
$$

The function $I_{0}(s, b, \xi)$ satisfies to the following sum rule

$$
\int I_{0}\left(s, b, y, p_{\perp}\right) p_{\perp} d p_{\perp} d y=\bar{n}(s, b) \operatorname{Im} U(s, b),
$$

where $\bar{n}(s, b)$ is the mean multiplicity depending on impact parameter. The "bare" flow $\bar{v}_{n}$ is related to the measured flow $v_{n}$ as follows

$$
v_{n}\left(s, b, y, p_{\perp}\right)=w(s, b) \bar{v}_{n}\left(s, b, y, p_{\perp}\right) .
$$


In the above formulas the variable $y$ denotes rapidity, i.e. $y=\sinh ^{-1}(p / m)$, where $p$ is a longitudinal momentum. Evidently, corrections due to unitarity are mostly important at small impact parameters, i.e. they provide an additional suppression of the anisotropic flows at small centralities, while very peripheral collisions are not affected by these corrections.

The geometrical picture of hadron collision at non-zero impact parameters described above implies that the generated massive virtual quarks in overlap region will obtain large initial orbital angular momentum at high energies. The total orbital angular momentum can be estimated as follows

$$
L(s, b) \simeq \alpha b \frac{\sqrt{s}}{2} D_{C}(b) .
$$

The parameter $\alpha$ is related to the fraction of the initial energy carried by the condensate clouds which goes to rotation of the quark system and the overlap region, which is described by the function $D_{C}(b)$, has an ellipsoidal form (Fig. 1). It

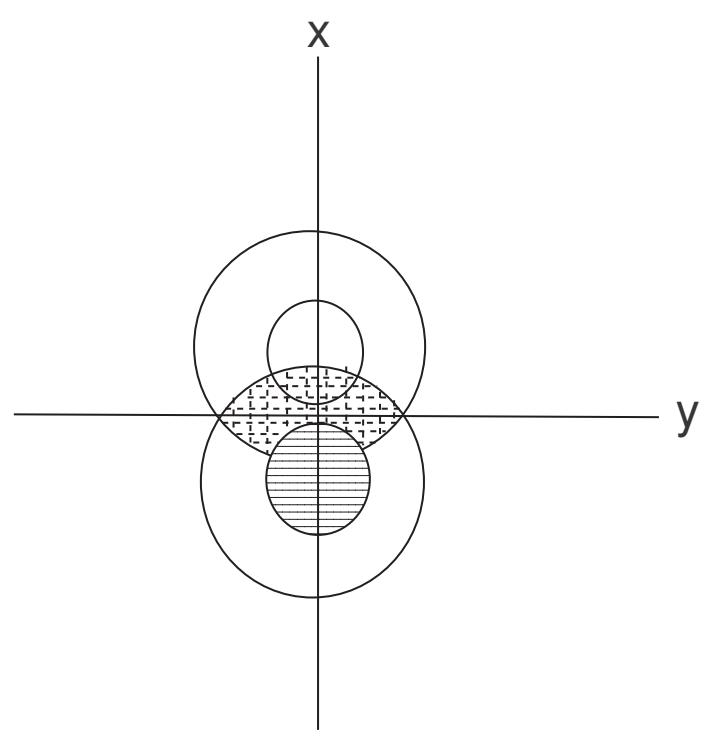

Figure 1: Schematic view in frontal plane of the hadron collision as extended objects. Collision occurs along the z-axis.

should be noted that $L \rightarrow 0$ at $b \rightarrow \infty$ and $L=0$ at $b=0$ (Fig. 2). 


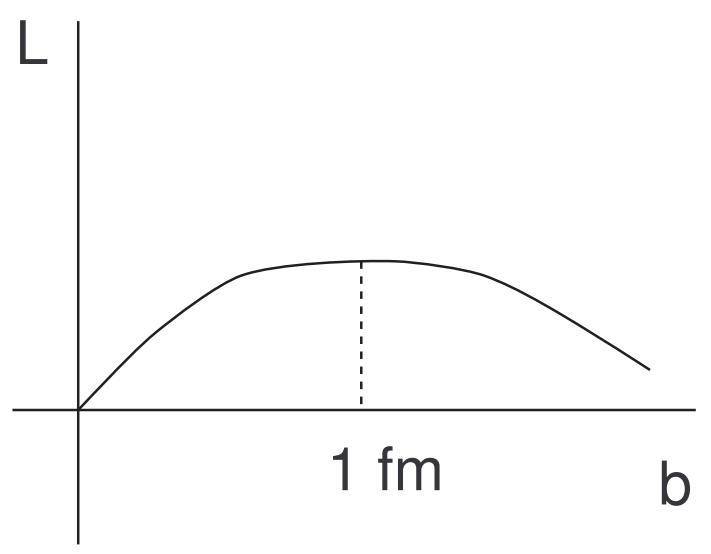

Figure 2: Qualitative dependence of the orbital angular momentum $L$ on the impact parameter $b$.

\section{Rotating transient matter and directed flow in hadronic reactions}

Now we would like to discuss the experimental consequences of the described picture of hadron collisions. The important problem here is the experimental determination of the impact parameter $\mathbf{b}$. To proceed that way the measurements of the characteristics of multiparticle production processes in hadronic collisions at fixed impact parameter should be performed with selection of the specific events sensitive to the value and direction of impact parameter. The determination of the reaction plane in the non-central hadronic collisions could be experimentally realizable with the utilization of the standard procedure[27, 28]. The relationship of the impact parameter with the final state multiplicity is a useful tool in these studies similar to the studies of the nuclei interactions. For example, in the Chou-Yang geometrical approach [29] one can restore the values of impact parameter from the charged particle multiplicity [30]. The centrality is determined by the fraction of the events with the largest number of produced particles which are registered by detectors (cf. [31]). Thus, the impact parameter can be determined through the centrality and then, e.g. directed flow, can be analyzed by selecting events in a specific centrality ranges. Indeed, the relation

$$
c(N) \simeq \frac{\pi b^{2}(N)}{\sigma_{\text {inel }}}
$$

between centrality and impact parameter was obtained [32] and can be extended straightforwardly to the case of hadron scattering. In this case we should consider $\bar{R}$ as a sum of the two radii of colliding hadrons and $\sigma_{\text {inel }}$ as the total inelastic 
hadron-hadron cross-section. The centrality $c(N)$ is the centrality of the events with the multiplicity larger than $N$ and $b(N)$ is the impact parameter where the mean multiplicity $\bar{n}(b)$ is equal to $N$.

At this point we would like to make an important remark. Namely, based on the recent discoveries at RHIC, we assume that the matter in the transient (intermidiate) state of hadronic reactions has a strongly interacting nature. Due to strong interaction between quarks in the transient state, it can be described as a liquid. Therefore, the orbital angular momentum $L$ should be realized as a coherent rotation of the quark-pion liquid as a whole in the $x z$-plane (due to mentioned strong correlations between particles presented in the liquid). It should be noted that for the given value of the orbital angular momentum $L$ kinetic energy has a minimal value if all parts of liquid rotates with the same angular velocity. We assume therefore that the different parts of the quark-pion liquid in the overlap region indeed have the same angular velocity $\omega$. In this model spin of the polarized hadrons has its origin in the rotation of matter hadrons consist of. In contrast, we assume rotation of the matter during intermediate, transient state of hadronic interaction.

Collective rotation of the strongly interacting system of the massive constituent quarks and pions is the main point of the proposed mechanism of the directed flow generation in hadronic and nuclei collisions. We concentrate on the effects of this rotation and consider directed flow for the constituent quarks supposing that directed flow for hadrons is close to the directed flow for the constituent quarks at least qualitatively.

The assumed particle production mechanism at moderate transverse momenta is an excitation of a part of the rotating transient state of massive constituent quarks (interacting by pion exchanges) by the one of the valence constituent quarks with subsequent hadronization of the quark-pion liquid droplets. Due to the fact that the transient matter is strongly interacting, the excited parts should be located closely to the periphery of the rotating transient state otherwise absorption would not allow to quarks and pions to leave the region (quenching). The mechanism is sensitive to the particular rotation direction and the directed flow should have opposite signs for the particles in the fragmentation regions of the projectile and target respectively. It is evident that the effect of rotation (shift in $p_{x}$ value ) is most significant in the peripheral part of the rotating quark-pion liquid and is to be weaker in the less peripheral regions (rotation with the same angular velocity $\omega)$, i.e. the directed flow $v_{1}$ (averaged over all transverse momenta) should be proportional to the inverse depth $\Delta l$ where the excitation of the rotating quark-pion liquid takes place (Fig. 3) i.e.

$$
\left|v_{1}\right| \sim \frac{1}{\Delta l}
$$

In its turn, the length $\Delta l$ is related to the energy loss of constituent valence quark 


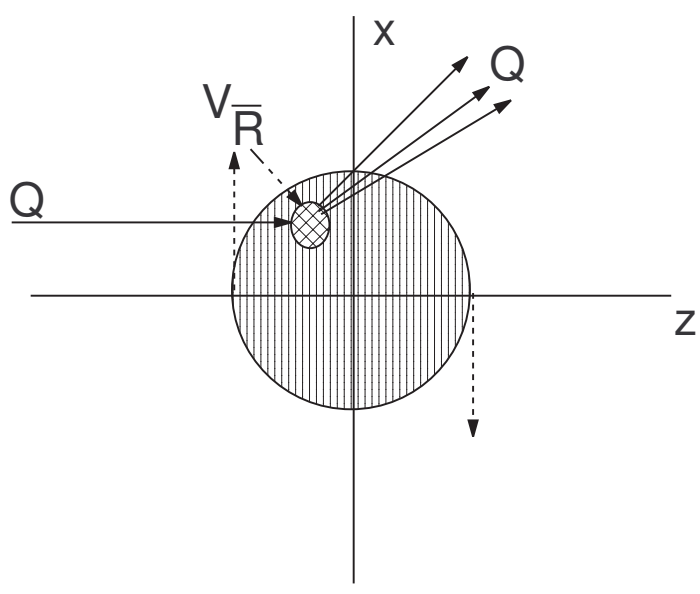

Figure 3: Interaction of the constituent quark with rotating quark-pion liquid.

in the medium due to elastic rescattering (quark-pion liquid) prior an excitation occurs, i.e. before constituent quark would deposit its energy into the energy of the excited quarks (those quarks lead to the production of the secondary particles), i.e. it can be assumed that

$$
\Delta l \sim \Delta y,
$$

where $\Delta y=\left|y-y_{\text {beam }}\right|$ is the difference between the rapidities of the final particle and the projectile. On the other hand, the depth length $\Delta l$ is determined by elastic quark scattering cross-section $\sigma$ and quark-pion liquid density $n$. Therefore, the averaged value of $v_{1}$ should be proportional to the particle density of the transient state and include cross-section $\sigma$, i.e.

$$
\left\langle\left|v_{1}\right|\right\rangle \sim \sigma n
$$

This estimate shows that the magnitude of the directed flow could provide information on the properties of the transient state. The magnitude of observable $v_{1}$ (determined by the shift of transverse momentum due to rotation) is proportional to $(\Delta l)^{-1}$ in this mechanism and depends on the rapidity difference as

$$
\left|v_{1}\right| \sim \frac{1}{\left|y-y_{\text {beam }}\right|}
$$

and does not depend on the incident energy. Evidently, the directed flow $\left|v_{1}\right|$ decreases when the absolute value of the above difference increases, i.e. $\left|v_{1}\right|$ increases at fixed energy and increasing rapidity of final particle and it decreases at fixed rapidity of final particle and increasing beam energy. Dependence of $\left|v_{1}\right|$ will be universal for different energies when plotted against the difference $\left|y-y_{\text {beam }}\right|$ (Fig.4). 


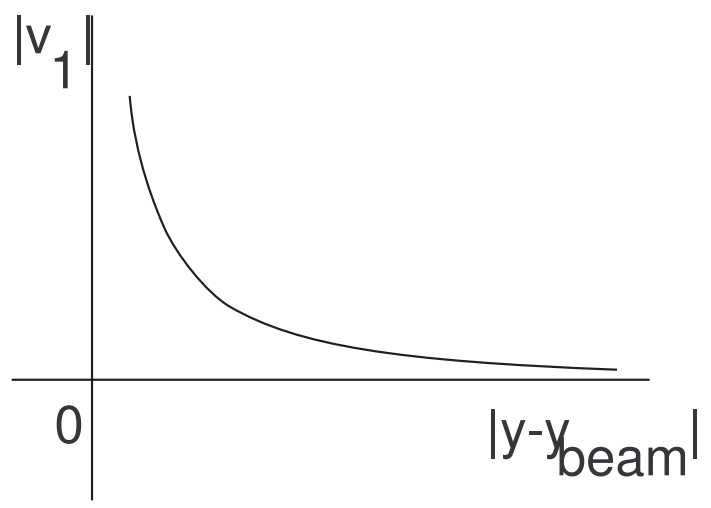

Figure 4: Universal dependence of directed flow on the rapidity difference.

The centrality dependence of $\left|v_{1}\right|$ is determined by the orbital momentum dependence $L$ on the impact parameter, i.e. it should be decreasing towards high and lower centralities. Decrease toward high centralities is evident, no overlap of hadrons or nuclei should be at high enough impact parameters. Decrease of $v_{1}$ toward lower centralities is specific prediction of the proposed mechanism based on rotation since central collisions with smaller impact parameters would lead to slower rotation or its complete absence in the head-on collisions. Thus, the qualitative centrality dependence of $\left|v_{1}\right|$ corresponds to Fig. 2.

Now we can consider transverse momentum dependence of the directed flow $v_{1}\left(p_{\perp}\right)$ (integrated over rapidity) for constituent quarks. It is natural to suppose that the size of the region where the virtual massive quark $Q$ comes from the quark-pion liquid is determined by its transverse momentum, i.e. $\bar{R} \simeq 1 / p_{\perp}$. However, it is evident that $\bar{R}$ should not be larger than the interaction radius of the valence constituent quark $R_{Q}$ (interacting with the quarks and pions from the transient liquid state). The production processes with high transverse momentum such that $\bar{R}$ is much less than the geometrical size of the valence constituent quark $r_{Q}$ resolve its internal structure as a cluster of the non-interacting partons. Thus, at high transverse momenta the constituent quarks will be excited themselves and hadronization of the uncorrelated partons would lead to the secondary particles with high transverse momenta and vanishing directed flow. If the production mechanism rendering to the constituent quark excitation is valid, then similar conclusions on the small anisotropic flows at large transverse momenta should be applicable for $v_{n}\left(p_{\perp}\right)$ with $n>1$. Obviously, it should not be valid in the case of the polarized hadron collisions.

\footnotetext{
${ }^{2}$ Of course, there is another reason for vanishing $v_{1}$ at $b=0$, it is rotational invariance around collision axis.
} 
The magnitude of the quark interaction radius $R_{Q}$ can be taken from the analysis of elastic scattering [18]; it has dependence on its mass in the form (4) with $\kappa \simeq 2$, i.e. $R_{Q} \simeq 1 \mathrm{fm}$, while the geometrical radius of quark $r_{Q}$ is about 0.2 $\mathrm{fm}$. The size of the region 4 , which is responsible for the small- $p_{\perp}$ hadron production, is large, valence constituent quark excites rotating cloud of quarks with various values and directions of their momenta in that case. Effect of rotation will be averaged over the volume $V_{\bar{R}}$ and therefore $\left\langle\Delta p_{x}\right\rangle_{V_{\bar{R}}}$ and $v_{1}\left(p_{\perp}\right)$ should be small.

When we proceed to the region of higher values of $p_{\perp}^{Q}$, the radius $\bar{R}$ is decreasing and the effect of rotation becomes more prominent, valence quark excites now the region where most of the quarks move coherently in the same direction with approximately equal velocities. The mean value $\left\langle\Delta p_{x}\right\rangle_{V_{\bar{R}}}$ and the directed flow, respectively, can have a significant magnitude and increase with increasing $p_{\perp}$. When $\bar{R}$ becomes smaller than the geometrical radius of constituent quark, the interactions at short distances start to resolve its internal structure as an uncorrelated cloud of partons. The production of the hadrons at such high values of transverse momenta is due to the excitation of the constituent quarks themselves and subsequent hadronization of the partons. The collective effects of rotating transient cloud in $v_{1}$ at large $p_{\perp}^{Q}>1 / r_{Q}$ will disappear as well as the directed flow of final particles. The value of transverse momentum, where the maximal values in the $p_{\perp}$-dependence of $v_{1}$ are expected, are in the region $1 \mathrm{GeV} / \mathrm{c}$ since $r_{Q} \simeq 0.2 \mathrm{fm}$. The qualitative $p_{\perp}$-dependence of the directed flow is illustrated on Fig. 5.

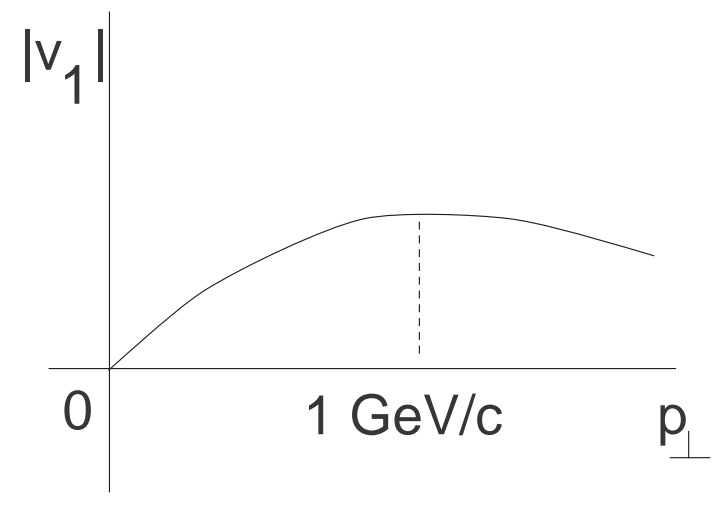

Figure 5: Qualitative dependence of directed flow on transverse momentum $p_{\perp}$.

\footnotetext{
${ }^{3}$ This is the light constituent quark interaction radius which is close to the inverse pion mass.

${ }^{4}$ For simplicity we suppose that this region has a spherically symmetrical form
} 


\section{Directed flow in nucleus collisions}

Until now we considered hadron scattering and directed flow in this process, but significant ingredient in this consideration was borrowed from the $A A$ studies, namely we supposed that the transient matter is a strongly interacting one and it is the same in $p p$ and $A A$ reactions. Using the experimental findings of RHIC, we assumed,in fact, that it is a liquid consisting of massive quarks, interacting by pion exchange, and characterized by the fixed interparticle distances determined by the quark interaction radius. The assumption on the almost instantaneous, shockwave type of generation of the transient state obtains then support in the very short thermalization time revealed in heavy-ion collisions at RHIC [33]. Existence of the massive quark matter in the stage preceding hadronization seems to be supported also by the experimental data obtained at CERN SPS [34].

The geometrical picture of hadron collision has an apparent analogy with collisions of nuclei and it should be noted that the appearance of large orbital angular momentum should be expected in the overlap region in the non-central nuclei collisions. And then due to strongly interacting nature of the transient matter we assume that this orbital angular momentum realized as a coherent rotation of liquid. Thus, it seems that underlying dynamics could be similar to the dynamics of the directed flow in hadron collisions.

We can go further and extend the production mechanism from hadron to nucleus case also. This extension cannot be straightforward. First, there will be no unitarity corrections for the anisotropic flows and instead of valence constituent quarks, as a projectile we should consider nucleons, which would excite rotating quark liquid. Of course, those differences will result in significantly higher values of directed flow. But, the general trends in its dependence on the collision energy, rapidity of the detected particle and transverse momentum, should be the same. In particular, the directed flow in nuclei collisions as well as in hadron reactions will depend on the rapidity difference $y-y_{\text {beam }}$ and not on the incident energy. The mechanism therefore can provide a qualitative explanation of the incident-energy scaling of $v_{1}$ observed at RHIC [35]. In the projectile frame the directed flow has the same values for the different initial energies. In the Fig. 6 experimental data are shown along with the dependence

$$
v_{1} \sim\left(\eta-y_{\text {beam }}\right)^{-1},
$$

where $\eta$ is a pseudorapidity. This dependence reflects the trend in the experimental data and, therefore, the mechanism described in the previous section obtains an experimental justification, qualitative, of course. In addition, we would like to note that azimuthal dependence of the suppression factor $R_{A A}$ found in the experiment PHENIX at RHIC [36] can also be explained by rotation of the transient 


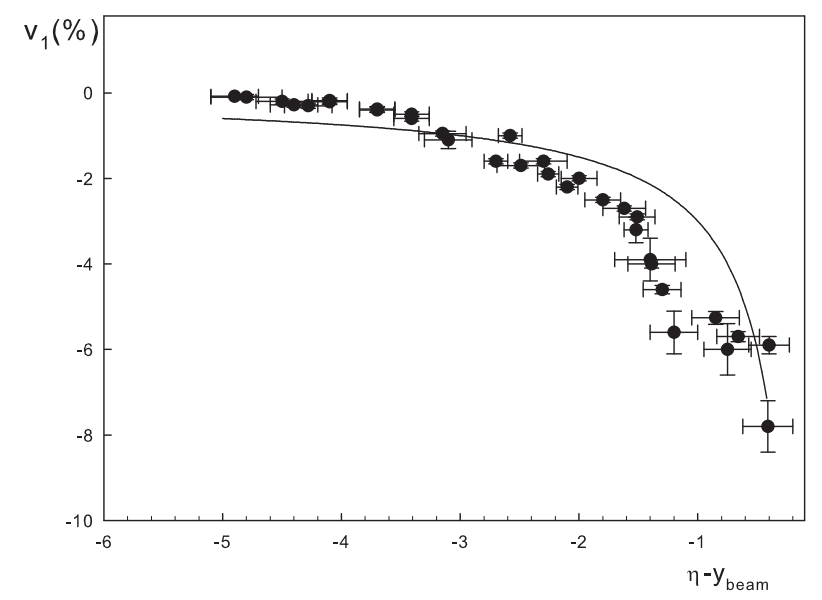

Figure 6: Dependence of directed flow on $\eta-y_{\text {beam }}$ in $A u+A u$ and $C u+$ $\mathrm{Cu}$ collisions at $62.4 \mathrm{GeV}$ and $200 \mathrm{GeV}$ at RHIC (preliminary data of STAR Collaboration [35])

state. Since the correlations result from the rotation in this mechanism and therefore are maximal in the rotation plane, similar dependence should be observed in the azimuthal dependence of the two-particle correlation function. Effect of rotation should be maximal for the peripheral collisions and therefore the dependence on $\phi$ should be most steep at larger impact parameter (or centrality) values The discussed rotation mechanism should contribute to the elliptic flow too. However, since the regularities already found experimentally for $v_{1}$ and $v_{2}$ in nuclei interactions imply different dynamical origin for these flows, we should conclude that this mechanism does not provide significant contribution to the elliptic flow.

\section{Directed flow at the LHC energies and conclusion}

Nowadays, with approaching start of the LHC, it is interesting to predict what should be expected at such very high energies [37], in particular would deconfined matter produced in $p p$ and $A A$ collisions be weakly interacting or it will remain to be a strongly interacting one as it was observed at RHIC in $A A$ collisions? In the latter case one can expect that proposed rotating mechanism will be working at the LHC energies and therefore the observed at RHIC incident-energy scaling in $v_{1}$ will remain to be valid also, i.e. directed flow plotted against the difference $y-y_{\text {beam }}$ will be the same as it is at RHIC. However, if the transient matter at the LHC energies will be weakly interacting, then one should expect absence of the coherent rotation and the vanishing of the directed flow. This conclusion is valid 
provided that the rotation is the only mechanism of the directed flow generation. If it is so, vanishing directed flow can serve as a signal of a genuine quark-gluon plasma (gas of free quarks and gluons) formation.

The parton interaction and finite transverse gradient of parton longitudinal momentum is a driving force of the orbital angular momentum conversion to the global system polarization through spin-orbital coupling [38]. In the parton picture, which is a weakly interacting medium, the coherent rotation is absent. The predicted global polarization was not found experimentally at RHIC [39] and it is just another, indirect confirmation of the strongly interacting character of the deconfined matter at such energies. It however does not mean that at the LHC energies picture will be the same. Thus, if the matter produced at the LHC is weakly interacting and since the total angular momentum should be conserved in any case, the orbital momentum would be converted then into global polarization at the partonic level which can be detected experimentally measuring hyperon or photon polarizations [38, 40]. It should be noted that there is another reason for the emerging global polarization of the secondary particles in hadron collisions at the LHC energies - it is antishadowing or reflective scattering [26, 41]. The latter mechanism is not related to the question of what kind of deconfined matter was produced.

Thus, it would be interesting to perform the measurements of the anisotropic flows at RHIC and at the LHC not only in heavy ion collisions, but in $p p$-collisions also, and to find possible existence or absence of the rotation effects related to strong interactions through the directed flow studies. At RHIC there is another possibility of the directed flow measurements in the polarized proton collisions. Those studies when combined with the measurements of the directed flow in the unpolarized $p p$-scattering, could provide information on the role of the orbital angular momentum in the spin structure of the nucleon. At large transverse momenta, they will provide information on the generalized parton distributions in the impact parameter space, which are under active studies nowadays (cf. [42]).

We discussed qualitative features of the transient state in hadronic and nuclei collisions related to the directed flow $v_{1}$. We concentrated on the hadron interactions. However, the most conclusions should remain to be valid for nucleus interactions.

\section{Acknowledgement}

We are grateful to J. Dunlop, V. Pantuev and O. Teryaev for the interesting discussions. We would like to acknowledge also valuable remarks and comments from the Referees and the Editorial board. 


\section{References}

[1] Quark Gluon Plasma. New Discoveries at RHIC: A Case of Strongly Interacting Quark Gluon Plasma. Proceedings, RBRC Workshop, Brookhaven, Upton, USA, May 14-15, 2004: D. Rischke, G. Levin, eds; 2005, 169pp; J. Adams et al. (STAR Collaboration) Nucl. Phys. A 757 (2005)102; K. Adcox et al (PHENIX Collaboration), Nucl. Phys. A 757 (2005) 184.

[2] D. Miśkoviec, [arXiv: 0707.0923].

[3] D. d'Enterria, [arXiv: nucl-ex/0611012].

[4] A. Peshier, W Cassing, Phys. Rev. Lett., 94 (2005) 172301;

M. Bluhm, B. Kämpfer, G. Soff, J. Phys. G 31 (2005) S1151;

J. Liao, E.V. Shuryak, hep-ph/0508035, 2005;

G.E. Brown, B.A. Gelman, M. Rho, Phys. Rev. Lett. 96 (2006) 132301

the most recent review is E. V. Shuryak, [arXiv: hep-ph/0608177].

[5] J. Hofmann, H. Stöcker, U. W. Heinz, W. Scheid, W. Greiner, Phys. Rev. Lett. 36 (1976) 88.

[6] H. Stöcker, J. A. Maruhn, W. Greiner, Phys. Lett. B 81 (1979) 303.

[7] H. Stöcker, J. A. Maruhn, W. Greiner, Phys. Rev. Lett. 44 (1980) 725.

[8] H. Stöcker, W. Greiner, Phys. Rept. 137 (1986) 277.

[9] H. Sorge, Phys. Rev. Lett. 82 (1999) 2048 [arXiv:nucl-th/9812057].

[10] M. Bleicher, H. Stöcker, Phys. Lett. B 526 (2002) 309 [arXiv:hep-ph/0006147].

[11] H. Stöcker, Nucl. Phys. A 750 (2005) 121 [arXiv:nucl-th/0406018].

[12] X. 1. Zhu, M. Bleicher, H. Stöcker, J. Phys. G 32 (2006) 2181 [arXiv:nucl-th/0601049].

[13] H. Petersen, Q. Li, X. Zhu, M. Bleicher, Phys. Rev. C 74 (2006) 064908 [arXiv:hep-ph/0608189].

[14] D. Molnar, and S.A. Voloshin, Phys. Rev. Lett. 91 (2003) 092301

S.A. Voloshin, Acta Physica Polonica B 36 (2005) 551;

[15] R.M. Weiner, Int. J. Mod. Phys. E 15 (2005) 37.

[16] J.-Y. Ollitrault, Phys. Rev. D46 (1992) 229; recent review can be found in S.A. Voloshin, Nucl. Phys. A715 (2003) 379.

[17] D. Molnar, [arXiv: nucl-th/0408044].

[18] S.M. Troshin, N.E.Tyurin, Phys. Rev. D 49 (1994) 4427. 
[19] S.M. Troshin, N.E. Tyurin, J. Phys. G 29, (2003) 1061.

[20] J.D. Bjorken, Nucl. Phys. Proc. Supl. 25B (1992) 253.

[21] D. Diakonov, [arXiv: hep-ph/0406043], JLAB-THY-04-12, Eur. Phys. J. A 24 (2005) 3;

D. Diakonov, V. Petrov, Phys. Lett. B 147 (1984) 351.

[22] T. Goldman, R.W. Haymaker, Phys. Rev. D24 (1981) 724.

[23] W. Heisenberg, Z. Phys. 133 (1952) 65.

[24] P. Carruthers, Nucl. Phys. A 418 (1984) 501.

[25] A.A. Logunov, V.I. Savrin, N.E. Tyurin, O.A. Khrustalev, Teor. Mat. Fiz. 6 (1971) 157.

[26] S.M. Troshin, N.E. Tyurin, Int. J. Mod. Phys. A 22 (2007) 4437.

[27] S.A. Voloshin, [arXiv: nucl-th/0410089].

[28] S.A. Voloshin, A.M. Poskanzer, Phys. Lett. B 474 (2000) 27.

[29] T.T. Chou, C.-N. Yang, International Journal of Modern Physics A, 6 (1987) 1727; Adv. Ser. Direct. High Energy Phys. 2 (1988) 510.

[30] T.T. Chou, C.N. Yang, Phys. Lett. 128B (1983) 457; Phys. Rev. D, 32 (1985) 1692.

[31] F. Antinori, Proc. of the XXXII International Symposium on Multiparticle Dynamics, Alushta, Crimea, Ukraine, September 2002, Eds. A. Sissakian, G. Kozlov, E. Kolganova, 77.

[32] W. Broniowski, W. Florkowski, Phys. Rev. C 65 (2002) 024905.

[33] K. Adcox, et al., Nucl. Phys. A757 (2005) 184;

J. Castillo (for the STAR Collaboration), Int. J. Mod. Phys. A20 (2005) 4380.

[34] J. Zimányi, P. Lévai, T.S. Biró, Heavy Ion Phys. 17 (2003) 205; J. Phys. G31, (2005) 711.

[35] G. Wang (for the STAR Collaboration), J. Phys. G 34 (2007) S1093, [arXiv: nucl-ex/0701045;

B.B. Back et al., (PHOBOS Collaboration), Phys. Rev. Lett., 97 (2006) 012301;

A.H. Tang (for the STAR collaboration), J. Phys. G 31 (2005) S35;

J. Adams et al. (STAR Collaboration), Phys. Rev. C 73 (2006) 034903.

[36] V.S. Pantuev, arXiv:hep-ph/0604268].

[37] S. Abreu et al., [arXiv:0711.0974]. 
[38] Z.-T. Liang, X.-N. Wang, Phys. Rev. Lett. 94 (2005) 102301, ibid. 96 (2006) 039901.

[39] B.I. Abelev et al, (STAR Collaboration), [arXiv:0705.1691].

[40] A. Ipp, A. Di Piazza, J. Evers, C.H. Keitel, [arXiv:0710.5700].

[41] S.M. Troshin, Phys. Lett. B 597 (2004) 391.

[42] M. Burkardt, Int. J. Mod. Phys. A 18 (2003) 173. 